EL PROCESO EN LA CIUDAD. EL CASO DEL TRASLADO DEL MERCADO NACIONAL DE HACIENDA DE LINIERS DURANTE LA ÚLTIMA DICTADURA MILITAR ARGENTINA

\title{
Luján Menazzi
}

Licenciada en Sociología por la Universidad de Buenos Aires y doctoranda en Ciencias Sociales en la misma universidad. Es docente en la Facultad de Ciencias de Sociales (UBA), becaria doctoral del CONICET con sede en el Instituto de Investigaciones Gino Germani (FSOC-UBA), donde además se desempeña en el Área de Estudios Urbanos como integrante del proyecto "Renovación y cambio en el sur de la ciudad de Buenos Aires", a cargo de la Dra. Hilda Herzer. Actualmente investiga sobre procesos urbanos en el barrio de Mataderos.1menazzi@yahoo.com.ar.

ISNN 1666-6I86. Volumen 12 No 12 (Junio 2012) pp. 095-II4 - Recibido: 09-II-II Aprobado: 04-04-I2 


\title{
Resumen
}

El artículo aborda las intervenciones y procesos urbanos iniciados durante la última dictadura a partir de un caso específico: el intento frustrado de trasladar el Mercado de Hacienda fuera de la ciudad de Buenos Aires. Se pretende reconstruir la dinámica político-institucional de este proceso atendiendo a cómo se tomó la decisión política y a los factores que incidieron en frustrar el traslado. A partir del análisis del caso, se busca relativizar ciertos balances que conceptualizan a la dictadura como un punto de quiebre total en términos de políticas urbanas, asignándole un carácter todopoderoso, premeditado y coherente. En esta línea se señalan para este caso dos cuestiones. Por un lado las distintas áreas encargadas de intervenir sobre lo urbano y la heterogeneidad de figuras en el interior del elenco dictatorial que defendían intereses diversos, lo cual tuvo como correlato una transformación muy parcial y poco coherente del área. En segundo término la capacidad de resistencia que pusieron de manifiesto ciertos sectores vinculados con el quehacer del mercado, entorpeciendo la voluntad política de trasladarlo.

$\underline{\text { Palabras clave }}$

Procesos urbanos, dictadura, Ciudad de Buenos Aires.

\begin{abstract}
The article analyses interventions and urban processes initiated during the last military dictatorship (1976-1983) on the basis of a specific case: the failed attempt to move the Cattle Market outside the city of Buenos Aires. The aim is to reconstruct the political dynamics of this process, considering the political decision and the factors that prevented the eradication. This paper seeks to call into question certain statements that conceptualize the dictatorship as a total turning point in terms of urban policy, considering it all-powerful, deliberate and consistent. With this purpose the paper delves into two issues related to this case. On the one hand the different government areas responsible for intervening in urban issues and on the other, the heterogeneity of high ranking figures within the dictatorial cast defending different interests. The first issue entailed a very partial and inconsistent transformation of the area. Secondly, the resilience shown by some sectors/actors in hindering the political will to move the market.
\end{abstract}

\section{Keywords}

Urban processes, dictatorship, Buenos Aires City. 
El proceso en la ciudad. El caso del traslado del Mercado Nacional de Hacienda de Liniers durante la última dictadura militar argentina

\section{INTRODUCCIÓN}

El presente trabajo se enmarca en la investigación realizada para la tesis doctoral "Procesos urbanos en Mataderos. Transformaciones y persistencias en un barrio industrial (19762006)". ${ }^{1}$ La tesis se propone analizar los modos en los que se conforma el espacio urbano de la Ciudad de Buenos Aires atendiendo a las maneras en que es configurado y reconfigurado por una articulación de procesos económicos, políticos, sociales y urbanos y por una amplia variedad de actores locales que desarrollan lógicas, dinámicas y temporalidades heterogéneas. Con este objetivo, el análisis focaliza en la proyección, transformación y persistencias de un territorio acotado del barrio de Mataderos. Para abordar esta problemática se sostienen dos escalas de análisis en paralelo. Por un lado, se trabajan las políticas urbanas a lo largo del período de análisis y los modos en que fueron cambiando los actores gubernamentales y locales intervinientes, los modos de intervención, las prioridades a la hora de intervenir, entre otras cuestiones. En paralelo y en diálogo con la mirada más general sobre los procesos y políticas urbanas, se analizan en particular dos intervenciones urbanas: en primer lugar los frustrados intentos por parte del poder político y organizaciones barriales de trasladar el MNH por fuera de los límites de la capital en diversos contextos político-institucionales $(1977,1986,2001)$; en segundo término el desmantelamiento del predio que fuera el Frigorífico Lisandro de la Torre y la posterior ocupación del terreno por la empresa Roemmers y el Parque Alberdi.

Este artículo se focaliza en el primer período que aborda la tesis, analizando las políticas urbanas de la última dictadura militar para la ciudad de Buenos Aires a partir de un caso en particular, el intento de trasladar el Mercado Nacional de Hacienda de Liniers (MNH) por fuera de los límites de la ciudad. Para ello, se retoman ciertas lecturas acerca de la dictadura que pretenden complejizar los balances tradicionales. Así, retomando la mirada político institucional del régimen que plantea CANELO (2008a, 2008b) se discutirán para el caso de las políticas urbanas y para el tema del $\mathrm{MNH}$ en particular dos cuestiones relacionadas: por un lado, la idea del régimen militar como un poder monolítico y absoluto, sin fricciones internas y, por otra parte, la mirada sobre el régimen como dotado de una coherencia ideológica y una voluntad teleológica de cumplir ciertos objetivos en áreas diversas. Para el caso de las políticas urbanas, la variedad y diversidad de intervenciones y la multiplicidad de actores estatales intervinientes, con lógicas diversas, suponen un impacto importante en el período dictatorial sobre la ciudad, pero no homogéneo. La mirada atenta a la dinámica político-institucional evidencia que "antes que planes políticos diseñados por los diversos protagonistas hay que pensar en términos de lógicas de acción diferenciadas" (QuiroGA,
1- Doctorado en Ciencias Sociales de la Universidad de Buenos Aires. 
${ }^{2}$ Existen discusiones similares respecto de la dictadura en términos de continuidad y ruptura en otras áreas. Un ejemplo de ello es el balance respecto de las políticas económicas. Así, frente a las primeras tesis de los años 80 , que sostienen un agotamiento del modelo de acumulación por sustitución de importaciones previo a la gestión de Martínez de Hoz (Müller y Rapetti, 2001), se han realizado numerosos análisis económicos del período que divergen profundamente, postulando no un agotamiento, sino un quiebre del nacional desarrollismo por parte de la gestión de Martínez de Hoz Canitrot, 1980: Schvarzer, 1983, Basualdo, 2006). Dentro de esta línea hay quienes interpretan ese quiebre con matices diversos, dando cuenta no solo de las rupturas, sino de ciertas líneas de continuidad con el período anterior (Pucciarelli, 2004, Castellani, 2009, Müller y Rapetti, 2001). Estos autores, además, complejizan y matizan las políticas económicas, dando cuenta de los vaivenes $e$ incongruencias de la batería de reformas económicas en diálogo con la coyuntura política.
2004: 83) que en muchas oportunidades resultarán confusas, ambiguas y contradictorias. Como veremos más adelante, para la dimensión urbana, por un lado existen ciertas miradas que entienden a la dictadura como un punto de quiebre total y absoluto respecto de las políticas previas; por el otro, existen lecturas que ven en la dictadura una articulación de continuidad (y culminación) de la tradición modernizadora-planificadora. En este sentido, resulta útil retomar perspectivas como las de QuIROGA (2004) y VEZZETTI (2002), quienes además de "pensar a la dictadura militar en clave política" (CANELO, 2008: 16) hacen énfasis en colocar a la dictadura en la trama de sentido de la historia argentina, y no como algo desvinculado de ella. En términos de VEZzETTI (2002), la última dictadura fue tanto una irrupción como un desenlace, y siguiendo a QuiRoGA (2004), el proceso adquiere sentido en la propia lógica del sistema político argentino.

Desde esta perspectiva se repasarán a continuación algunos abordajes a las políticas urbanas de esa etapa señalando ciertos matices en la conceptualización de los distintos autores. En esta línea se destacarán particularmente aquellos autores que señalan las continuidades y rupturas del período analizado respeto de períodos precedentes ${ }^{2}$. En segundo término, se pretende reconstruir la dinámica político-institucional del intento de trasladar el MNH por fuera de la ciudad de Buenos Aires atendiendo, en primer lugar, al modo en que se tomó la decisión política de efectuar el traslado y, en segundo lugar, a los factores que incidieron en que el traslado no se produzca, particularmente, la oposición de ciertos actores privados interesados en que el MNH permanezca en la capital. Las reflexiones finales buscan poner en diálogo las teorizaciones sobre las políticas urbanas del período y el análisis del caso en particular y realizar algunos señalamientos a partir de su desarrollo posterior.

\section{POLÍTICAS URBANAS EN DICTADURA: BALANCES Y PERSPECTIVAS}

Existe un acuerdo generalizado en el campo de los estudios urbanos de que en las últimas décadas la ciudad de Buenos Aires ha sufrido profundas transformaciones. Esta percepción se condice con un proceso internacional de transformación de las ciudades, la entrada en crisis de un ciclo "expansivo" o "desarrollista" a partir de procesos como la deslocalización industrial, el fin del crecimiento demográfico urbano por migraciones, la proliferación de espacios insulares, las críticas al urbanismo moderno y la emergencia de nuevas formas de intervenir en la ciudad (PAndo, Gorelik, et ál., 2004; Novick, 2003).

A partir de este acuerdo básico se presentan diversas lecturas en cuanto al carácter y profundidad de estas transformaciones y el momento en que comenzaron. En este marco,

Cuaderno Urbano. Espacio, Cultura, Sociedad - Vol. 12 - No 12 (Junio 20I2)- Pp. 095-II4 - ISNNI666-6186 


\section{El proceso en la ciudad. El caso del traslado del Mercado Nacional de Hacienda de} Liniers durante la última dictadura militar argentina los procesos urbanos iniciados en la dictadura militar o los efectos urbanos de muchas políticas de la dictadura son interpretados por muchos autores como un parte aguas en varios sentidos respecto de las formas de intervenir previamente en la ciudad. Poniendo en diálogo a autores que se enfocan en diversos objetos de estudio, pero que coinciden en analizar algunos aspectos del período de la dictadura, se advierten ciertos matices. Así el análisis de OszlaK (1991) pone el énfasis en que es a partir de la dictadura cuando se pone en vigencia una nueva jerarquía del espacio urbano, vinculado con la concepción del orden social que sostenía este sistema de gobierno, como uno de los grandes lineamientos políticos que organizaban medidas aparentemente aisladas y con diversos fines inmediatos. Por su parte LIERNUR (2001) también reconoce transformaciones clave en las temáticas, los modos de gestión, las características de la organización industrial y las condiciones de los encargos durante el período dictatorial, sin dejar de señalar que la dictadura funcionó como una culminación del período previo (con un Estado como promotor de grandes obras y planes, aunque en un sentido inverso al que hasta entonces sustentaba). ${ }^{3}$ TORREs (2006, 2001 ) establece como corte en su periodización la década de los 80 , indicando que en ese momento se iniciaron fuertes transformaciones, vinculadas con el deterioro central en Buenos Aires, y que en ese contexto de crisis comienzan a ensayarse nuevas estrategias de intervención en la ciudad. Destaca también que durante los 90, estos procesos de deterioro se acentúan drásticamente (Torres, 2001). Domínguez RocA (2005), por su parte, observa que las políticas urbanas de la dictadura articularon elementos que podrían considerarse contradictorios, en tanto algunos presentaban líneas de continuidad con planes y propuestas previas y otros elementos marcaban fuertes diferencias con las ideas previas. Por otra parte, para SILVESTRI y GORELIK (2000) la ruptura más profunda respecto de las tradiciones urbanas anteriores se produce recién en la década del 90, que habría implicado una nueva configuración del espacio urbano, mientras que en 1976 y en 1984, fechas que supusieron quiebres políticos centrales al iniciarse en 1976 la dictadura militar y en 1984 el retorno democrático, se habrían producido desplazamientos o rupturas de menor alcance.

Las divergencias se vinculan en parte con los distintos focos de interés de estos autores, pero también con los modos en que plantean los vínculos entre procesos sociales, políticos, económicos y urbanos y el grado de autonomía o importancia relativa que se le da a cada uno. Así, SiLVESTRI (2002) y LiERNUR (2001) resaltan fuertemente las relaciones ambiguas y tangenciales de los procesos políticos y las formas arquitectónicas, cuestionando la supeditación lineal de hechos urbanos a cambios políticos y económicos, mientras que TORREs (1993) destaca el carácter estrecho e interactivo de la relación entre procesos de estructuración espacial y los procesos económicos, sociales y políticos. Otros autores, a
3- Es importante aclarar que la periodización de Liernur responde a criterios disciplinares de la propia arquitectura y no a criterios politicos. 
$\overline{4-\text { Las diferentes fuentes }}$ utilizadas en estos trabajos también dan cuenta de la diversidad de perspectivas $y$ focos de interés. Así, si Torres analiza la configuración metropolitana a partir de los censos, Liernur se focaliza en las obras de arquitectura más relevantes de cada período y Oszlak en el desarrollo de procesos politico-institucionales en contextos autoritarios.

5- Esta enumeración de obras no es exhaustiva, e incluye tanto intervenciones municipales como de diversas reparticiones del gobierno nacional. su vez, periodizan los cambios en la ciudad según procesos políticos locales (FERNÁNDEZ WAGNER, 2008) o ponen el foco en procesos económicos transnacionales (CicCOLELLA, 1999). Difieren entonces no solo los objetos de estudio, sino las perspectivas respecto de los vínculos entre las dimensiones que inciden en lo urbano. ${ }^{4}$

Lo que resulta indudable, más allá del tenor y del sentido de las transformaciones urbanas iniciadas de modo directo o indirecto en el período, es el enorme impacto de la dictadura en la ciudad de Buenos Aires, la gran cantidad de intervenciones físicas y de reglamentaciones con fuertes efectos sobre el espacio urbano realizadas por la Municipalidad y por el gobierno nacional a través de diversas reparticiones. Por mencionar solo algunas de las cuestiones más destacadas, podemos nombrar la construcción de gran cantidad de parques y plazas, la construcción de autopistas y las expropiaciones y demoliciones que supusieron, la construcción de equipamientos (terminal de Retiro, ATC, Centro Cultural Recoleta, las escuelas municipales, grandes conjuntos habitacionales), demolición y traslado de equipamiento considerado obsoleto o contaminante (frigoríficos, Hospital Salaberry, incineradoras de residuos, basurales), ensanche de avenidas, construcción de estacionamientos subterráneos, descentralización o traslado de servicios (CEAMSE, Mercado Central), expulsión de población de bajos recursos por medio de la liberalización de los alquileres y la erradicación violenta de villas de emergencia. ${ }^{5}$ Esto sin contar algunos proyectos truncos que de todas formas dejaron su marca en la ciudad, como el Ensanche del Área Central y la traza de la Autopista 3.

Un eje central de impacto fue sin dudas el Código de Planeamiento Urbano de 1977, que enmarcaba muchas de las intervenciones antes citadas. El código, en correlación con la reestructuración económica del período, busca el alejamiento del perfil industrial de la ciudad a través de la restricción de lo industrial a ciertas industrias y zonas muy específicas y una orientación hacia un perfil residencial (en términos restrictivos), de servicios y de negocios. Esta reorientación comienza a darse en el período y se profundiza con posterioridad (Oszlak, 1991; Ciccolella, 1999; Gorelik, 2004). Por medio del código se premiaba la construcción en perímetro libre permitiendo mayor altura y se limitaba la altura de los edificios entre medianeras, se proponía la construcción de un sistema de parques en consonancia con otras políticas ambientalistas, se alentaba la construcción de grandes equipamientos en el sur por medio del englobamiento de parcelas y se reglamentaba la expulsión de ciertos usos industriales considerados nocivos para el área urbana (por ejemplo la prohibición de faenar ganado en la ciudad, lo que implicaba la expulsión de mataderos $\mathrm{y}$, eventualmente de frigoríficos y la prohibición de incinerar basura). 


\section{El proceso en la ciudad. El caso del traslado del Mercado Nacional de Hacienda de}

Liniers durante la última dictadura militar argentina
Repasando estas intervenciones sobre la ciudad, nos encontramos con un conjunto ecléctico. En términos de PANDO, GoRELIK et ál. (2004) el período de la dictadura se caracterizó por conjugar lineamientos que continuaban con el período expansivo y otros elementos que se insertaban en los lineamientos posexpansivos, aspectos que en alguna medida podrían considerarse contradictorios (Domínguez RocA, 2005). Así, muchas de las iniciativas de Cacciatore $^{6}$ se encuadran en la tradición modernizadora-planificadora, retomando planes y proyectos previos: el código de planeamiento, la zonificación, la construcción de autopistas, la construcción de escuelas y espacios verdes (PANDO, GoRELiK, et ál.; 2004). Al mismo tiempo, muchas políticas con efectos urbanos se vinculaban, por el contrario, con el decaimiento de ese ciclo expansivo y el comienzo de nuevas preocupaciones y perspectivas: los procesos de desindustralización de la ciudad, las políticas económicas de desincentivo a la actividad industrial y crecimiento del sector terciario, la preocupación por la calidad ambiental, los incipientes avances de lo patrimonial, el énfasis en la ciudad como espacio residencial para los sectores de mayores ingresos y la consiguiente expulsión de sectores populares a través de mecanismos diversos.

La diversidad de los procesos urbanos llevados adelante por la dictadura militar, sostenidos por miradas distintas y en ocasiones contradictorias en torno a la ciudad, se vincula con varias cuestiones. Por un lado, la heterogeneidad que caracterizó al gobierno de la última dictadura militar en tanto en su interior convivían funcionarios de diversas ideologías y tradiciones. En términos de CANELO (2008) "muy lejos de encarnar un poder monolítico, la alianza cívico-militar que encabezó el Proceso fue un conjunto heterogéneo y conflictivo" (CANELO, 2008: 17). Es importante destacar que, como en cualquier otro gobierno, la dictadura se conformaba por pensamientos heterogéneos, con fuertes contradicciones internas y pujas por la toma de decisiones. Esta característica se manifiesta también en las decisiones en torno a los procesos urbanos, que tienen ciertas características específicas. En primer lugar, el cruce de lógicas políticas contradictorias y las internas burocráticas cobran particular visibilidad en lo urbano, ya que no solo las políticas específicamente urbanas tienen efectos territoriales, sino también, y con particular fuerza, ciertas medidas económicas y ciertas políticas sociales ${ }^{7}$ Vinculada con esto, se suma la superposición de entes estatales de distintos niveles que inciden sobre lo urbano, con lógicas diversas. A su vez, para el tema específico de los procesos urbanos, es importante considerar las temporalidades extensas de ciertos procesos que involucran a la ciudad. SILVESTRI y GoRELIK (2000) afirman que "... la ciudad, por su carácter de artefacto material, somete a las dimensiones sociales, políticas y culturales a la prueba de la larga duración...” (SILVESTRI y GoRELIK, 2000: 461). Los proyectos urbanos, los reglamentos que rigen el funcionamiento y los usos
6- Osvaldo Cacciatore fue el intendente de facto de la Municipalidad de Buenos Aires entre el 2 de abril de 1976 y 31 de marzo de 1982 Perteneciente a la aviación militar, participó en 1951 del intento de golpe liderado por Menéndez contra el gobierno de Juan Domingo Perón y en el bombardeo a la Plaza de Mayo en 1955. Su gestión a cargo de la ciudad se caracterizó por la realización de obras consideradas faraónicas y por la expulsión de población de bajos recursos de la ciudad de Buenos Aires a través de diversas iniciativas, como la erradicación de villas, la expulsión de actividades industriales y las expropiaciones por construcción de autopistas.

7- En términos de Torres (2006), se trata de politicas urbanas implícitas, aquellas políticas que no están formuladas con fines urbanos pero que tienen gran impacto sobre la ciudad. 
$\overline{\text { 8- El MNH es el ente encar- }}$ gado de centralizar la compra y venta de ganado. Hoy en día, su participación en la comercialización del ganado ha descendido mucho, pero aún sigue funcionando como precio de referencia. El MNH está ubicado en el barrio de Mataderos, ocupaba 40 hectáreas en el período de análisis y hoy ocupa 32.

9- Arturo Frondizi fue presidente de la República Argentina entre 1958 y 1962, año en que fue derrocado por un golpe militar. La intención de trasladar y privatizar el MNH fue manifestada en el Decreto 7.793 del 4/9/61, que indicaba que la Comisión Administradora del Mercado debía estudiar la conveniencia del traslado de las instalaciones así como el traspaso del organismo a la actividad privada. La medida puede vincularse con la privatización del Frigorífico Lisandro de la Torre, llevada adelante dos años antes por el mismo gobierno. del espacio, la disposición física de la ciudad son elementos que exigen un período muy extenso para poder modificarse. En este sentido se encuadran los vínculos de la dictadura con tradiciones que la precedían.

Lo que quizá sí represente una marca propia de la dictadura fue la capacidad de concretar numerosas obras de carácter faraónico en la ciudad. Esto se vincula con el contexto dictatorial como facilitador para llevar adelante procesos políticos complejos, de gran magnitud, en tanto se eludían los consensos imprescindibles en un régimen democrático y se anulaban oposiciones que en otras coyunturas políticas habrían tenido mayor peso, poder y voz. Esto se relaciona a su vez con una fuerte voluntad de acción transformadora respecto del espacio urbano, voluntad que en ocasiones priorizaba las obras concretas por sobre los planes a largo plazo (Domínguez RocA, 2005). Así como en las políticas económicas, las intervenciones urbanas del período buscaron transformaciones drásticas e irreversibles. Las intervenciones urbanas se articulaban de forma coherente con una búsqueda más profunda y general del gobierno dictatorial: la búsqueda del orden (OszLaK 1991).

\section{A continuación se abordará el intento de trasladar del MNH en el contexto de la}

\section{dictadura militar.}

El MNH ocupaba una superficie de 40 hectáreas en el barrio de Mataderos, ubicado en el sector sudoeste de la ciudad de Buenos Aires. Su eventual traslado implicaba grandes transformaciones urbanas para la ciudad en su conjunto. El tema estaba atravesado, a su vez, no solo por inquietudes ligadas a lo urbano, sino, principalmente, por criterios económicos acerca de la industria de la carne. En este sentido, el ente político encargado de decidir los destinos del MNH era el Ministerio de Economía, y al interior de este, la Secretaría de Agricultura.

\section{EL TRASLADO DEL MNH EN EL CONTEXTO DE LA ÚLTIMA DICTADURA}

El 29 de diciembre de 1976, cuando todavía se transitaba el primer año de la dictadura militar, se decide crear por resolución de la Secretaría de Agricultura (que dependía en ese entonces del Ministerio de Economía), la Comisión de Traslado del $\mathrm{MNH}^{8}{ }^{8}$ El tema del traslado del MNH y su privatización ya había sido planteado previamente, en 1961, durante el gobierno de Arturo Frondizi'; sin embargo, no se había avanzado al respecto. Para 1976, la idea de trasladar el MNH cobra fuerza en el contexto de la dictadura en coherencia con intereses de las diversas esferas del gobierno dictatorial. Por un lado el deseo de racionalizar, liquidar o reducir ciertos entes estatales vinculados con políticas previas de control e intervención por parte del Estado. Vinculado con esto, el deseo de descentralizar ciertas 
El proceso en la ciudad. El caso del traslado del Mercado Nacional de Hacienda de Liniers durante la última dictadura militar argentina actividades consideradas perniciosas para la ciudad de Buenos Aires. ${ }^{10}$ Por otra parte, la voluntad de desarrollar en términos urbanísticos de diversos modos a la ciudad de Buenos Aires y, a su vez, las preocupaciones ambientales respecto de la ciudad que buscaban entre otras cuestiones deshacerse de viejos equipamientos urbanos considerados anacrónicos y contaminantes. También en términos de la política dirigida hacia el sector del comercio de carnes, se buscaba liberalizar y racionalizar, con lo cual la continuidad de funcionamiento del MNH y su traslado a un emplazamiento más adecuado en términos ambientales y más avanzados en términos técnicos era coherente. ${ }^{11}$ El tema está atravesado, a su vez, por el hecho de que Videla ${ }^{12}$ incentivaba particularmente el traslado en tanto este se realizaría al partido de Mercedes ${ }^{13}$, su lugar de nacimiento, y consideraba que la instalación del MNH beneficiaría económicamente a la zona. Este último factor era el motor central e inicial para que el traslado se produzca. La gestión fue encarada por voluntad de Videla, y no era un tema prioritario a los ojos del secretario de Agricultura del momento, más interesado en la liberalización del comercio exterior y del sistema cambiario para los comercios del agro y en desmantelar los antiguos frigoríficos. En este marco de confluencia de varios intereses, el traslado del MNH resultaba un proceso lógico para los lineamientos políticos de la dictadura, tanto a nivel económico como político y urbano.

A fines de 1976 se crea la Comisión de Estudio del Traslado del MNH con el objetivo de analizar el posible traslado y decidir el destino adecuado para el MNH. La comisión, encargada de llevar adelante la primera etapa del proceso de traslado, se conformó por representantes de los distintos actores involucrados en el tema del $\mathrm{MNH}$ : los consignatarios de hacienda, la industria frigorífica, los productores de hacienda, el gobierno de la provincia de Buenos Aires y la Secretaría de Agricultura de Nación.

Pocos meses después, en mayo de 1977, esta comisión decide oficialmente, pese a las fuertes discrepancias en su interior, que el destino para el nuevo MNH sea la localidad de Mercedes; sin embargo, como lo mencionáramos anteriormente, el destino ya estaba decidido de antemano por el interés del Gral. Videla en el tema. Esta decisión preestablecida se mantuvo no obstante las manifiestas divergencias que existían entre los integrantes de la comisión, ya que todos los que representaban al sector privado se oponían a la localización elegida.

\section{LA DISPUTA EN TORNO AL DESTINO DEL MNH}

A partir de ese momento, comenzó una disputa que se hizo pública a través de los medios de comunicación en torno al posible destino del MNH. Los diversos actores privados in-
10- Un ejemplo muy vinculado con esto sería el traslado del Mercado Central a Tapiales iniciado previamente y materializado por la dictadura.

11- Según Cadenas Madariaga y Carmelo Pafundi (secretario de Agricultura y director del MNH en esos momentos el funcionamiento del $M N H$ era sumamente eficiente, su función era central y no implicaba ninguna erogación para el Estado, puesto que se sostenía con sus propios recursos (entrevista a Carmelo Pafundi, 2/2010, y a Cadenas Madariaga, 3/2010).

12- Jorge Rafael Videla fue uno de los líderes del golpe de Estado del 24 de marzo de 1976 que derrocó al gobierno de Isabel Martínez de Perón e instauró el autodenominado Proceso de Reorganización Nacional. Hasta ese momento se desempeñaba como teniente general de las Fuerzas Armadas. Presidió de facto la República Argentina entre el 29 de marzo de 1976 y el 29 de marzo de 1981, instaurando el régimen más sangriento que gobernó el país.

13- Se trata de un partido de la provincia de Buenos Aires ubicado a 100 kilómetros al oeste de la ciudad de Buenos Aires. 
14- El Centro de Consignatarios es una entidad centenaria, que agrupa casas consignatarias encargadas de comercializar la hacienda. Se trata de casas pertenecientes a familias muy tradicionales y poderosas que se dedican a esta actividad desde mediados del siglo XIX. Un repaso por los nombres de algunas de estas casas resultará ilustrativo: Mendizábal; Aberasturi: Aguirre Urreta; Alzaga Unzué; Sáenz Valiente; Harrington y Lafuente; Heguy; Lacoste; Lalor; Lanusse Santillán; Bullrich; Lynch; Santamarina. Las casas llevan como nombre los apellidos de familias tradicionales y poderosas, ligadas con la actividad agropecuaria.

15- En el diario Clarín del 8 de octubre de 1977 se publica la nota "El nuevo Liniers estará a $100 \mathrm{~km}$ de la Capital". En esa nota se publica la opinión contraria de los consignatarios. En La Campaña del 14 de octubre de 1977 se publican las declaraciones de los consignatarios en la nota "El Gobierno debería tomar represalias contra los países de la comunidad", y en La Prensa se publican varias notas que difunden la oposición al proyecto por parte de los consignatarios, como por ejemplo el 31 de diciembre de 1977, "Ezeiza sería la zona ideal para ubicar el Mercado de Hacienda”. volucrados en la dinámica del MNH manifestaron públicamente su oposición a través de extensas notas aparecidas en diversos medios. En el lapso de una semana aparecen notas en el diario La Prensa del Centro de Consignatarios de Productos del País ${ }^{14}$, de la Asociación de Propietarios de Camiones de Transporte de Hacienda en pie y de la Cámara Argentina de la Industria Frigorífica manifestando su oposición al traslado del MNH a la zona de Mercedes por la excesiva distancia y sugiriendo una zona más cercana a la capital. Las tres entidades coincidían en sugerir al partido Ezeiza como el destino ideal, por su justa distancia y por la red de infraestructura que se estaba desarrollando debido a la instalación en aquella zona del Mercado de Concentración Frutihortícola. El Centro de Consignatarios era el organismo que con más fuerza se oponía al traslado y comenzó una fuerte campaña en contra que se extendió a lo largo de todo el proceso.

A pesar de estas manifestaciones de oposición a la localización elegida, el 22 de septiembre de 1977 se aprueba el traslado a la localidad de Mercedes por medio del Decreto 2891. Se justifica por "necesidades impostergables de salubridad de la ciudad de Buenos Aires, así como también su desarrollo urbanístico". Por medio del mismo decreto se conforma una nueva comisión, la Comisión de Traslado del MNH, presidida por el subsecretario de ganadería de la Secretaría de Agricultura y cuyos miembros serían un coordinador de la secretaría de Agricultura, un representante de la Comisión Administradora del MNH, uno de la Junta Nacional de Carnes, uno del Centro de Consignatarios, uno del Servicio de Sanidad Animal, uno del Gobierno de la Provincia de Buenos Aires y uno de la Municipalidad de Mercedes. La comisión debería encargarse de seleccionar las tierras para el nuevo $\mathrm{MNH}$, programar y llevar adelante el traslado y proyectar la venta de los terrenos en Mataderos, teniendo en cuenta la opinión de la Municipalidad de la Ciudad de Buenos Aires. Con la venta de los terrenos se planeaba financiar los gastos de la nueva radicación y construcción. Los gastos del funcionamiento de la comisión debían ser cubiertos por los fondos del MNH. Una vez realizado el anuncio oficial, se congregó público a festejar la noticia frente a la Municipalidad de Mercedes.

Aun con el decreto firmado, el Centro de Consignatarios de Hacienda no dio por cerrado el tema. A través de un fluido contacto con todos los medios de comunicación e interpelando a los responsables gubernamentales, la medida fue discutida públicamente con virulencia. Inmediatamente después de anunciado el decreto salieron notas contrarias al traslado por parte del Centro de Consignatarios en diversos medios de comunicación (diario Clarín, diario La Campaña). ${ }^{15}$ A su vez, a través de los diarios La Prensa, La Campaña, La Nación y El Orden (diario de Mercedes) se produjo una fortísima polémica entre el Centro de 


\section{El proceso en la ciudad. El caso del traslado del Mercado Nacional de Hacienda de} Liniers durante la última dictadura militar argentina
Consignatarios y el intendente de Mercedes, con acusaciones cruzadas entre ambos por supuestos "intereses sectoriales" de un lado e "intereses localistas" del otro. ${ }^{16}$

En febrero de 1978 el Centro de Consignatarios, la Cámara Argentina de la Industria Frigorífica, la Cámara Argentina de Frigoríficos, Industriales y Exportadores de Carne, la Asociación de Industrias Argentinas de Carnes y la Asociación de Propietarios de Camiones del Transporte de hacienda en pie le escribieron una nota al presidente Videla. La nota expresa la fuerte oposición de estas entidades al traslado, afirmando que "los mercados, como el de Liniers, no se forman por decreto". A su vez, ponían en duda el éxito del nuevo MNH insinuando que los compradores y los vendedores no asistirían allí: "¿Se justifica imponer semejante esfuerzo al erario público, ante la evidente desproporción que existe entre la inversión a realizar y la remota posibilidad de lograrse que el Mercado funcione a pleno en Mercedes? ¿Si el nuevo mercado fracasa como tal por insuficiente concurrencia de usuarios, reconocerán su responsabilidad quienes estimulan el traslado del actual ente nacional a dicha zona?". El tono de la nota era muy crítico. Por un lado se afirmaba que el MNH no iba a funcionar en su nueva localización, por otra parte se apuntaba contra quienes incitaban el traslado a Mercedes. La nota recomendaba fuertemente también la privatización del $\mathrm{MNH}$, en tanto esto sería acorde con la filosofía de privatizaciones encarada por el gobierno. Los cuestionamientos a la medida oficial se siguieron manifestando con la misma virulencia mientras se tuvo la intención de hacer cumplir la medida.

Por su parte, a fines de enero de 1978 la comisión conformada para llevar adelante el traslado estaba trabajando al respecto, teniendo ya seleccionada una ubicación para el MNH en Mercedes y presentando los decretos complementarios para llevar adelante el traslado. A principios de septiembre de 1978 se emite la Ley 21.872 , por la cual se declaran de utilidad pública y sujetos a expropiación terrenos de 200 hectáreas en el partido de Mercedes, a los fines de radicar allí al MNH. Los terrenos fueron sugeridos por la comisión, considerando su accesibilidad, su cercanía a los ferrocarriles y red caminera. Los gastos debían quedar a cargo del $\mathrm{MNH}$.

Una vez comenzadas las gestiones para llevar adelante el traslado, y antes de comenzar las expropiaciones, el asunto se volvió problemático por varias cuestiones. Por un lado, los terrenos elegidos constituían un loteo urbano materializado, debiéndose expropiar mil quinientas parcelas. Esto resultaba muy oneroso económicamente y problemático en términos operativos, en tanto había que lidiar con numerosos propietarios y en muchos casos había inconvenientes con los títulos de propiedad. En este sentido era preferible optar por
16- La polémica llegó a incluir la convocatoria por parte del intendente de Mercedes a las fuerzas vivas de la ciudad para discutir y apoyar el tema del traslado $y$ los graves insultos de los consignatarios. Algunos de los artículos de diario al respecto: 16 de diciembre de 1977 el Semanario Rural publica "Nueva sede del Mercado Nacional de Hacienda", el 3 de enero de 1978 el diario El Orden publica "Replica el intendente manifestaciones tendenciosas", el 9 de enero de 1978 en La Prensa aparece la nota "Traslado del mercado nacional de hacienda a la ciudad de Mercedes: el 16 de enero de 1978 en el mismo diario: "Acerca del traslado del Mercado de Liniers". 
17- Las innumerables obras encaradas por la Municipalidad de Buenos Aires en ese periodo fueron todas por adjudicación directa. También las importantes obras encaradas por el gobierno nacional con miras al Mundial 78 se realizaron por encargo directo. Esto implicó la ruptura con la tradición de los concursos que caracterizaba al periodo previo (Liernur, 2001; Silvestri y Gorelik, 2000). terrenos rurales, con otros valores de suelo y viabilidad operativa. Por otra parte, el MNH, encargado de solventar los gastos del traslado, carecía de los fondos para hacerlo.

Estos problemas supusieron una importante demora en el avance del traslado. En junio de 1980, se derogó finalmente la Ley 21.872, que indicaba la expropiación de los terrenos, y se promulgó una nueva, la Ley 22.236 que expropiaba otros terrenos en Mercedes, próximos a los seleccionados previamente, pero de tipo rural. Se trataba de tan solo cinco parcelas que sumaban algo más de 200 hectáreas. Estas pertenecían a cuatro propietarios, con las obvias ventajas que esto suponía. A su vez la ley cambia el origen del financiamiento, que ya no sería dado por MNH sino por Rentas Generales. La ley sugiere que una vez construido el nuevo MNH se consideraría su traspaso a manos privadas. Por otra parte, se afirma que el traslado "permitirá desarrollar edilicia, económica y socialmente una importante zona de la Ciudad de Buenos Aires, al par que la venta de los terrenos e instalaciones del citado Mercado constituirán un ingreso considerable para el Tesoro Nacional”.

En paralelo, el Código de Planeamiento sancionado a comienzos de 1977 por la Municipalidad asignaba el terreno que quedaría vacante por el traslado del MNH y por el cierre del Frigorífico Lisandro de la Torre para desarrollo urbanístico industrial, destinado a la relocalización de industrias y depósitos de usos "no conformes" en otras zonas de la ciudad. El resto del barrio era designado como área industrial o de grandes equipamientos, exceptuando pequeños sectores destinados a uso residencial.

A su vez, a partir de una iniciativa del Ministerio de Cultura y Educación, se declara Monumento Histórico al antiguo edificio de Administración de los Mataderos, en mayo de 1979, en el primer y único gesto de resguardo por el destino de los terrenos que quedarían vacantes en la ciudad de Buenos Aires.

\section{EL CONCURSO Y EL FRENO}

El 24 de septiembre de 1980 se abre el Concurso de Anteproyectos para la Construcción del Mercado de Concentración de Ganado en Mercedes, siendo uno de los pocos concursos de anteproyectos del período dictatorial. ${ }^{17}$ Tan solo unos días después de la apertura del concurso y con la voluntad de garantizar que el proyecto se iba a materializar, el 28 de septiembre se coloca la piedra basal del nuevo MNH con la presencia del presidente Videla, Zorreguieta como nuevo Secretario de Agricultura, Martínez de Hoz y el entonces gobernador de la provincia de Buenos Aires, Ibérico Saint Jean. El gesto pretendía dar cuenta 


\section{El proceso en la ciudad. El caso del traslado del Mercado Nacional de Hacienda de}

Liniers durante la última dictadura militar argentina de la voluntad inalterable de que el traslado se produzca. A su vez, la piedra se colocaba como parte de los festejos del aniversario de la fundación de la ciudad.

El 10 de febrero de 1981, el jurado conformado por el subsecretario de Ganadería, representantes de la Federación Argentina de Sociedades de Arquitectos y de la Sociedad Central de Arquitectos, un representante del Centro Argentino de Ingenieros, un representante del SENASA y representantes de la Secretaría de Agricultura da su fallo. Entre los representantes de la Secretaría de Agricultura está Roberto Arancedo, quien era a su vez el vicepresidente del Centro de Consignatarios del País y una figura clave en la disputa contra el traslado del MNH. El primer premio fue para el proyecto de dos estudios de importante trayectoria en obra pública, que se asociaron para este concurso: Estudio Staff y Aftalion, Bischoff, Egozcue, Vidal arqs.

El jurado seleccionó el proyecto por la solvencia técnica que presentó, la zonificación funcional y clara y el sistema circulatorio, entre otras cuestiones. Sin embargo, sectores de consignatarios y posteriormente, algunos integrantes de reparticiones estatales lo caracterizaron como faraónico e inviable. ${ }^{18}$

Una vez realizado el concurso y elegido el proyecto ganador a principios de 1981, se frenó el proceso de traslado. En el marco de cierta búsqueda de austeridad económica, se comenzó a replantear la financiación. Hay quienes decían, incluso desde la esfera gubernamental, que el proyecto del MNH debería esperar unos años para poder ser realizado. También se planteó la concesión a privados de la obra, porque se asumía que el Estado no estaba en condiciones de realizarla. Por último, se empezó a plantear con fuerza que el MNH mismo se privatice y que el privado se hiciera cargo de la construcción del nuevo $\mathrm{MNH}$, sea en Mercedes o en otra ubicación. A su vez, se comienza a afirmar que "la obra que se realice sea absolutamente antitética con el proyecto faraónico que se había propuesto" (La Nación 30/1/82). El gradual abandono del proyecto se vincula fuertemente con un cambio de etapa del régimen. En términos de Quiroga (2006), se trata de modificaciones reales en el rumbo del proceso, realineamientos en las relaciones de fuerzas (articulando cuestiones políticas y económicas). Estos realineamientos se vinculaban por un lado con el cambio en la Presidencia que implicó el recrudecimiento de las diferencias entre diversas fracciones de las Fuerzas Armadas. Las diferencias se vinculaban entre otras cuestiones con los modos de concebir el vínculo con la sociedad civil y con las miradas sobre la política económica (CANElo, 2008a). En términos de Quiroga (2006) esta etapa podría caracterizarse como de agotamiento del régimen, vinculado con la profunda crisis económica iniciada en 1980 y
18- Un representante de consignatarios señaló que el proyecto iba a ser muy costoso, en tanto se proponían cuestiones como techar el área de los corrales, que resultaba innecesario y oneroso. En esta línea señaló que el proyecto era ridículo (entrevista con dirigente del Centro de Consignatarios de Hacienda, 5 y 6/2010). A su vez, en el diario La Nación se publica un editorial en el que un funcionario tilda al proyecto de faraónico (La Nación, 30 de enero de 1982). 
19- Entrevista a Carmelo Pafundi, 2/2010.

20- Entrevista con un dirigente del Centro de Consignatarios (5 y 6/2010).

21- Entrevista con Fernando Aftalión (5/2010).

22- Estos vínculos y presiones de actores privados con el Estado podrían ser pensados, siguiendo la tipificación construida por Castellani (2009) como prácticas institucionalizadas, prácticas de influencia directa y prácticas de colonización de algunos reductos de la administración pública. la pérdida de legitimidad que comenzó a sufrir a partir de 1978. El abandono de grandes proyectos que involucraban ingentes fondos públicos o deuda pública puede observarse no solo en el caso analizado, sino también en las obras ligadas al sistema de autopistas de la ciudad de Buenos Aires, entre otras.

Los actores involucrados disienten respecto de los motivos por los cuales el traslado no progresó. Por un lado, hay quienes señalan que fue la oposición de los consignatarios la que logró frenar el traslado. En esta línea, el secretario de Agricultura del período afirma que el mayor inconveniente para afrontar el traslado fue la oposición de las casas consignatarias, por razones de comodidad. También el presidente del MNH en esa época señala que fueron los consignatarios los que lograron frenarlo. Para él, quien participó de todo el proceso desde adentro del MNH, la frustración del proyecto se debió al poder de los consignatarios, todos provenientes de familias tradicionales y poderosas y con fluidos contactos con el gobierno. Si bien les asigna la responsabilidad de frenar el traslado a estas "manos poderosas y ocultas", también señala que la inestabilidad política impedía encarar un proyecto de esa magnitud: "Cuando un gobierno se interesaba en seguida se tenía que ir". ${ }^{19}$ Siguiendo esta segunda línea y negando la posible responsabilidad de los consignatarios, un dirigente del Centro de Consignatarios ${ }^{20}$ señaló el cambio del momento político como el responsable de frustrar el proyecto. El proyecto habría caído solo, según este consignatario, por el cambio de la coyuntura política. Por último, uno de los integrantes del proyecto ganador señala a la incapacidad política para llevarlo adelante, subrayando que en este tipo de cuestiones primaba la corrupción y los caprichos de los políticos de turno por sobre el fundamento técnico. Si bien reconoce la fuerte oposición de los consignatarios, en su opinión estos no tenían ni el peso político ni económico para frenar el proyecto. ${ }^{21}$

Más allá de las diversas hipótesis, el análisis del proceso evidencia (sobre todo hacia el final de la dictadura) que existían diferencias en el interior del elenco gubernamental. Si bien había actores muy comprometidos con el traslado (como el propio Videla y el intendente de Mercedes), había otros que consideraban que esto no era prioritario (el secretario de Agricultura Cadenas Madariaga y figuras involucradas con el traslado, que participaban como representantes del gobierno pero formaban parte al mismo tiempo de las entidades que más se oponían). La ralentización de ciertos segmentos del proceso permite la sospecha de que existían trabas en el interior de las propias comisiones encargadas de llevar adelante las gestiones del traslado. ${ }^{22}$ Los desacuerdos en el interior del gobierno respecto del destino elegido, dimensiones y características del nuevo MNH se ponen de manifiesto una vez concluido el mandato de Videla, señalando la necesidad de privatizar el $\mathrm{MNH}$, la inviabilidad del proyecto y la posibilidad de proponer otro destino. 
Sin duda concurrieron varios factores para que el traslado no se produzca. Por un lado la falta de un consenso aglutinante a favor. Si bien todos los actores involucrados acordaban con la necesidad de trasladar el MNH, el destino no había sido concertado con los actores más relevantes del sector. Aunque se monto formalmente una serie de procesos en los que se contemplaba la participación de los actores más involucrados en el tema para que emitieran su opinión, la motivación principal para que el traslado se produzca a Mercedes implicaba que ciertas cuestiones no iban a ser discutidas ni negociadas. En esta línea, el traslado a Mercedes no respondía a una demanda de los actores involucrados, sino todo lo contrario. Por otra parte la magnitud misma del proceso, su complejidad y los tiempos largos que requería lo hacían más vulnerable al fracaso de presentarse oposiciones tenaces o discontinuidades en la voluntad gubernamental. Evidentemente la capacidad de los actores involucrados para hacerse oír y para interpelar las decisiones del gobierno dictatorial era alta, particularmente si se la compara con otros procesos acaecidos en la dictadura que involucraron el desplazamiento de actividades y de población fuera de la capital sin capacidad alguna de manifestar oposición. A su vez, que la motivación principal para efectuar el traslado fuera la voluntad personal presidencial lo hacía más vulnerable a los vaivenes políticos.

\section{REFLEXIONES FINALES}

El análisis del caso nos permite reflexionar acerca de algunas dimensiones de los procesos urbanos durante la dictadura. Poniéndolo en diálogo con las intervenciones urbanas del período, el caso tiene elementos que permiten su lectura en clave de continuidad con el período previo. Así, la voluntad de trasladar el MNH había sido planteada con anterioridad por parte del Estado nacional, buscando una ubicación más adecuada. Esta intervención se vinculaba con la idea de organización territorial de la ciudad, conduciendo su crecimiento y expansión y la reubicación de los servicios insalubres, teniendo al Estado como promotor y regulador de estas grandes obras. En diálogo con otras intervenciones y reglamentaciones urbanas del período también se podrían interpretar ciertos elementos (o cierto modo de plantearlos) como novedosos: la preocupación por la calidad ambiental, la incipiente búsqueda de participación de privados en el desarrollo urbano, la descentralización de servicios.

Resulta útil traer a colación la distinción realizada por ToRRES (2006) respecto de políticas urbanas implícitas y explícitas. En tanto políticas económicas de organización y renovación orientadas hacia muchas ramas de actividades productivas, el proceso analizado no constituiría una política urbana en sentido explícito, sino una política pública con importantes efectos urbanos, lo que TORRes (2006) llama política urbana implícita. Si bien la norma 
23-Además de los motivos no estrictamente urbanos, otros indicios que permiten interpretar a este caso como una política urbana implícita son la repartición estatal encargada de llevar adelante la medida (Secretaría de Agricultura) y la focalización de la medida en el nuevo $M N H$, no en los terrenos que quedarian vacantes.

24- I1, E2 y E3 son zonificaciones del Código de Planeamiento Urbano para industrias y grandes equipamientos. menciona al desarrollo urbano como uno de sus objetivos, está claro que no se trata de la motivación prioritaria para llevar adelante la medida. ${ }^{23}$ Por su parte, aquellas políticas urbanas explícitas llevadas adelante para el área no armonizaban enteramente con la política dispuesta a nivel nacional. Si a nivel nacional se proponía una renovación del área para su desarrollo urbanístico, el Código de Planeamiento no acompañaba esta propuesta de transformación de la zona y designaba para Mataderos una zonificación de Equipamientos e Industrias, continuando con el tradicional entramado industrial que existía en torno al MNH y proponiendo la ubicación de industrias de usos no conformes. Los alrededores del MNH y la mayor parte del barrio tiene zonificación I1, E2 y E3 ${ }^{24}$, lo cual da cuenta de que desde la órbita municipal no se pensaba en términos de transformación del área, sino más bien en términos de continuidad con lo que venía siendo. Sin embargo, una vez confirmada la medida de traslado del MNH, y realizada la liquidación y demolición del Frigorífico Lisandro de la Torre, que ocupaba grandes terrenos adjuntos al MNH, la municipalidad intenta reacomodarse respecto de esta suerte de renovación urbana que de modo directo o indirecto se impulsaba en el nivel nacional. Así, construye el Parque Alberdi en 1981 en parte del predio que antes fuera el frigorífico Lisandro de la Torre. El parque, contiguo al $\mathrm{MNH}$, fue construido explícitamente con la voluntad de acompañar el traslado del MNH y la consiguiente reconversión de la zona. A su vez, a nivel nacional pero desde otra repartición (Ministerio de Cultura y Educación) se declara Monumento Histórico al Antiguo Edificio de Administración de los Mataderos, resguardando una pequeñísima parte del MNH de su destino de venta a privados. Para el caso analizado, se podría pensar en términos de lo que TORREs (2006) llama las contradicciones entre las políticas urbanas implícitas y explícitas y la superposición de diversos entes estatales operando con lógicas distintas.

Otro punto interesante es la participación de los actores involucrados en el MNH. Por un lado, a diferencia de otros procesos que se desarrollaban paralelamente, el del traslado busca incorporar a los actores involucrados en el funcionamiento del MNH. Esto se debe a la jerarquía económica y social de estos actores y a que formaban parte del entramado que sostenía y apoyaba los lineamientos generales del régimen militar. Esta participación, sin embargo, tiene características formales, en tanto muchas de las decisiones en torno al traslado del MNH ya estaban tomadas antes de que se empezara a trabajar en las comisiones. Más allá de esto, se trató de actores con la capacidad de hacer oír su voz por medios privilegiados, interpelando al gobierno, obligándolo a salir al cruce de diversas críticas y justificar las decisiones adoptadas a través de aclaraciones, conferencias de prensa y actos de fuerte simbolismo. A su vez, existían ciertas divergencias al interior de las propias 
reparticiones estatales respecto del traslado del MNH. Estas divergencias se insinuaron durante el gobierno de Videla y se volvieron manifiestas con posterioridad. La participación de actores contrarios al traslado en la propia Secretaría de Ganadería y Agricultura y en las comisiones encargadas de motorizarlo permite suponer que ciertos retrasos y obstáculos no fueron enteramente involuntarios.

Reflexionando acerca de este proceso desde la actualidad se observan ciertas continuidades y rupturas en el tema: por un lado, los intentos para trasladar al MNH se multiplicaron a lo largo del tiempo, la voluntad estatal de trasladarlo continuó. Sin embargo, cambiaron los motores de ese traslado. Si en 1977 y posteriormente en 1986 fue el Estado nacional el que buscaba materializar el traslado, en 2001 fue la Ciudad Autónoma de Buenos Aires la principal interesada en impulsarlo. A su vez, si en 1977 y en 1986 se proyectó la construcción del nuevo $\mathrm{MNH}$, en 2001 se realiza un concurso de anteproyectos para darles destino a las tierras que quedarían vacantes una vez que el $\mathrm{MNH}$ se traslade, mientras que el proyecto para el nuevo MNH quedaría a cargo de la empresa concesionaria sin intervención alguna del Estado. Esto presenta marcados contrastes: la mirada del Estado local se centra en las tierras que hoy ocupa el MNH, proyectando diversos equipamientos y destinos para esos terrenos. La posibilidad de vender las tierras a privados no se plantea, como sí se había planteado en las normativas 1977 y 1986. Por otra parte la construcción del nuevo MNH pasa a ser una potestad de un grupo privado (los consignatarios de hacienda, que hoy en día están a cargo del MNH desde su privatización en 1992). Estos elementos dan cuenta de ciertas novedades a la hora de pensar los modos de intervenir en la ciudad. En primer lugar, la intervención activa de la Ciudad Autónoma de Buenos Aires como partícipe político con cierta autonomía implica la principal novedad y supone un cambio en el enfoque de la cuestión, valorizando el destino de las tierras de capital por sobre el destino del MNH. Por otra parte, aquellos privados que en 1977 y 1986 se opusieron al traslado hoy están a cargo de la concesión del MNH y tienen la potestad de decidir el destino de este y las características del nuevo predio. Por último si bien la actividad que centraliza el MNH pasó a privados, la idea de vender las tierras públicas fue relegada, lo cual supone una compleja redefinición de los límites y roles entre públicos y privados. 


\section{BIBLIOGRAFÍA}

BASUALDO, Eduardo (2006) Estudio de historia económica argentina desde mediados de siglo XX a la actualidad. Buenos Aires, FLACSO-Siglo XXI.

CANELO, Paula (2008a) El Proceso en su laberinto. La interna militar de Videla a Bignone. Prometeo, Buenos Aires.

CANELO, Paula (2008b) "Las "dos almas" del proceso. Nacionalistas y liberales durante la última dictadura militar argentina (1976-1981)" Páginas Revista Digital de la Escuela de Historia año $1, \mathrm{~N} .^{\circ} 1$, Rosario.

CANITROT, Adolfo (1980) "La disciplina como objetivo de la política económica" en Desarrollo Económico (76), Buenos Aires.

CASTELLANI, Ana (2009) Estado, empresas y empresarios. La difusión de ámbitos privilegiados de acumulación en la Argentina entre 1966 y 1989. Prometeo, Buenos Aires.

CICCOLELLA, Pablo (1999) "Globalización y dualización en la Región Metropolitana de Buenos Aires: grandes inversiones y reestructuración socioterritorial en los años noventa." En: EURE, Revista de Estudios Urbanos y Regionales, Vol. 25 N. 76 . Pontífica Universidad de Santiago de Chile, Santiago de Chile.

DOMÍNGUEZ ROCA, Luis (2005) "Planes urbanos y transporte en la Ciudad de Buenos Aires" En: Scripta Nova Revista electrónica de Geografia y Ciencias Sociales Vol. IX N. ${ }^{\circ}$ 194. Universidad de Barcelona, Barcelona.

FERNÁNDEZ WAGNER, Raúl (2008) Democracia y ciudad. Procesos y políticas urbanas en las ciudades argentinas (1983-2008). Universidad de General Sarmiento, Buenos Aires.

GORELIK, Adrián (2004) Miradas sobre Buenos Aires. Siglo XXI Editores Argentina, Buenos Aires.

LIERNUR, Francisco (2001) Arquitectura en la Argentina del siglo XX. La construcción de la modernidad. Fondo Nacional de las Artes, Buenos Aires.

MÜLLER, Alberto y RAPETTI Martín (2001) “ Un quiebre olvidado: la política económica de Martínez de Hoz" en Revista Ciclos N. ${ }^{\circ}$ 21, Buenos Aires.

NOVICK, Alicia (2003) "Espacios y proyectos. Oposiciones, hegemonías e interrogantes. En NOVICK, A. (ed.) Las dimensiones del espacio público. Problemas y proyectos. SPGCBA, Akian, Buenos Aires.

OSZLAK, Oscar (1991) Merecer la ciudad. Los pobres y el derecho al espacio urbano. Cedes-Humanitas, Buenos Aires.

PANDO, Horacio; GORELIK, Adrián; BALlenT, Anahí; AlIATA, Fernando; SILVESTRI, Graciela (2004). "Buenos Aires” En: LIERNUR, Francisco y ALIATA, 
Fernando (comps.): Diccionario de Arquitectura en la Argentina. Estilos, obras, biografias, instituciones, ciudades. AGEA, Buenos Aires.

PUCCIARELLI, Alfredo (2004). "La patria contratista. El nuevo discurso liberal de la dictadura militar encubre una vieja práctica corporativa". En: Alfredo Pucciarelli (coord): Empresarios tecnócratas y militares. Siglo XXI, Buenos Aires.

QUIROGA, Hugo (1990) El tiempo del Proceso. Conflictos y coincidencias entre políticos y militares. 1976-1983. Fundación Ross, Rosario.

SCHVARZER, Jorge (1983) Martínez de Hoz: La lógica política de la política económica, CISEA, Buenos Aires.

SILVESTRI Graciela y GORELIK Adrián (2000) "Ciudad y cultura urbana, 19761999: el fin de la expansión”, En: ROMERO, José Luis y ROMERO Luis Alberto (dir.), Buenos Aires, historia de cuatro siglos. Altamira, Buenos Aires.

SILVESTRI, Graciela (2002) “Apariencia y verdad. Reflexiones sobre obras, testimonios y documentos de arquitectura producidos durante la última dictadura", En: Block N. ${ }^{\circ} 5$, Buenos Aires.

TORRES, Horacio (2001) "Cambios socioterritoriales en Buenos Aires durante la década de 1990". En: EURE, Revista de Estudios Urbanos y Regionales, Vol. 2, ‥ ${ }^{\circ} 8$, Santiago de Chile.

TORRES, Horacio (2006) El Mapa Social de Buenos Aires (1940-1990). Edición Dirección de Investigaciones, Secretaría de investigación y Posgrado de la Facultad de Arquitectura/UBA, Buenos Aires.

VEZZETTI, Hugo (2002) Pasado y presente. Guerra, dictadura y sociedad en la Argentina. Siglo XXI,

Buenos Aires.

\section{FUENTES}

Diarios: LA PRENSA, LA NACIÓN, CLARÍN, LA CAMPAÑA, EL ORDEN.

Notas: CENTRO DE CONSIGNATARIOS DE HACIENDA a Aguado (presidente de CARBAP) 10/1977;

CENTRO DE CONSIGNATARIOS DE HACIENDA a Acuña (director del Semanario Rural) $12 / 1977$;

CENTRO DE CONSIGNATARIOS DE HACIENDA a Mihura (presidente de la Comisión de Traslado del MNH y Subsecretario de Ganadería) 1/1978.

CENTRO DE CONSIGNATARIOS DE HACIENDA a Videla (presidente de la Nación) 2/1978. 
CENTRO DE CONSIGNATARIOS DE HACIENDA al director del diario El Orden 3/1978.

CENTRO DE CONSIGNATARIOS DE HACIENDA a D'Apice (director del diario La Campaña) 9/1979.

CENTRO DE CONSIGNATARIOS DE HACIENDA a Aguado (ministro de Agricultura y Ganadería de la Nación) 10/1981.

\section{Legislación:}

ARGENTINA. PODER EJECUTIVO NACIONAL. Mercado Nacional de Hacienda. "Decreto Nacional N. ${ }^{\circ} 2891 "$ del 22 de septiembre de 1977. Buenos Aires, Boletín Oficial de la República Argentina, 1977.

Entrevistas: Carmelo Pafundi (director del Mercado de Hacienda entre 1966 y 1992) 2/2010.

Mario Cadenas Madariaga (secretario de Agricultura entre 1976 y 1979) 3/2010.

Fernando Aftalión (miembro del Equipo ganador del proyecto para el nuevo Mercado de Hacienda) 5/2010.

Dirigente del Centro de Consignatarios de Hacienda. 5 y 6/2010. 